\title{
Alternate Link Maximum Energy Level Ad Hoc Distance Vector Scheme for Energy Efficient Ad Hoc Networks Routing
}

\author{
Tai Hieng Tie ${ }^{1}$, Chong Eng Tan ${ }^{2}$, Sei Ping Lau ${ }^{3}$ \\ Department of Computer Systems and Communication Technologies \\ Faculty of Computer Science and Information Technology \\ University Malaysia Sarawak, \\ 94300 Kota Samarahan, Sarawak, Malaysia \\ tietaihieng@yahoo.co.uk ${ }^{1}$, cetan@fit.unimas.my ${ }^{2}$, splau@fit.unimas.my ${ }^{3}$
}

\begin{abstract}
Energy conservation is a very important design issue and has been attracting a lot of attention in recent years. The typical Mobile Ad Hoc Networks (MANET) routing protocols of the Internet Engineering Task Force (IETF) are mainly the shortest path routing protocols and do not consider the energy aware issue. This paper describes improvement of the conventional routing protocol by utilizing higher energy path and backup route. Our proposed algorithms adapt the existing AODV routing protocol to select the optimal route based on the basis of the maximum energy of each route. Computer simulations using ns-2 simulator are performed with comparison to the conventional methods and the effectiveness of the proposed methods is quantitatively validated.
\end{abstract}

Keywords-Energy Efficient Routing, Ad hoc Network

\section{INTRODUCTION}

In mobile ad hoc network, mobile nodes communicate with each other through wireless links without requiring existing network infrastructure. Nodes in the network do not depend on any infrastructure; mobile nodes establish dynamic, multi-hop network structure and set up communication links between devices if they lie within transmission range of each other. Due to the infrastructure-less characteristic, these kinds of networks have face many challenges such as unreliable links, lack of scalability, limited resources, etc. Most of the mobile communication devices in a wireless ad hoc network are powered by batteries. Batteries with limited energy have imposed a constraint on the entire MANET's performance. Strategies to save the node energy and prolong the lifetime of the network gradually plays an important role especially in the situation where temporary network connectivity is required such as in military areas, disaster areas, and conferences.

In view of the energy efficient routing, this paper proposes an energy efficiency routing protocol, namely the Alternate Route Maximum Energy Level Ad Hoc Distance
Vector (ALMEL-AODV) based on the basis of the classical routing protocol, the Ad hoc On-Demand Distance Vector (AODV) for Ad Hoc network. The ALMEL-AODV introduces maximum energy path on the network layer by combining remaining energy of all link nodes as an important parameter for route selection. The route with highest combined energy in the route table will be a better path for routing over the ad hoc network as the life span of the selected path can be extended to ensure maximum path availability for communication. If there are broken link, the next alternate route in the route table will be chosen to continue routing the packets to the destination.

\section{BACKGROUND}

In ad hoc network, all devices can communicate within or outside their radio range and they are self-organizing and adaptive. Routing protocols can be categorized into two categories: namely proactive and reactive. Proactive protocols maintain routes to all destinations at all times, regardless of whether they are needed or not. Such protocols have the advantage of minimal initial delay for communication with arbitrary destinations. On the other hand, reactive routing algorithms find a route only when desired by a source node. Under highly dynamic link conditions, the reactive protocols are expected to generate fewer overhead messages and provide a more reliable routing than the proactive routing protocols [1].

The AODV [2] is a basic reactive routing protocol with combination of Dynamic Source Routing (DSR) [3] and Destination Sequenced Distance Vector (DSDV) [4] routing protocols. It has the basic of the on demand mechanism of route discovery and route maintenance of DSR; and adopted the hop by hop routing mechanism via the sequence number and the periodic hello beacon of the DSDV. In the AODV 